\title{
Mitochrondrial therapy: A new concept for therapeutic management for tropical diseases
}

\section{Viroj Wiwanitkit* \\ Visiting Professor, Hainan Medical University, China}

Mitochondria is an important cellular organelle. This organelle plays important role regarding intracellular energy. Of interest, this organelle has its specific set of genetic components. At present, several medical disorders, such as Parkinson disease, are proven for their rooted causes as mitochondrial disorders [1]. In mitochondrial disease, mutations in genes in the mitochondrial DNA (mtDNA) that encode structural mitochondrial proteins or proteins involved in mitochondrial function is observed and this is the basic rooted pathogenesis of the clinical problem [1].

The mitochondrial disease becomes the new challenge in biomedicine. The current management of mitochondrial disease is still not conclusive. The advance mitochondrial therapy is the new therapeutic concept focusing at mitochondria. This therapy can be useful for management for not only specific mitochondrial disease but also other diseases. This approach has been proposed for a few years and noted for possible benefit in some medical problems such as sepsis [2]. Here, the authors would like to specific discuss on the use of mitochondrial therapy for the tropical medical disorders.

Indeed, using mitochondrial therapy might be useful for management of many tropical disorders. For example, mitochondria is proposed as a novel target for treatment of malaria [3]. In malariology, there are some new researches on this specific issue. Targeting at parasitic mitochondria is a new concept for management of drug resistant malaria [3]. Similarly, for dengue, another important mosquito borne infection, the role of mitochondrial is mentioned in the pathophysiology of the disease. In dengue, the virus can alter the mitochondrial membrane inducing cellular apoptosis and result in clinical abnormality [4]. The manipulation of the mitochondria in dengue becomes the new interesting research issue. The focus on mitochondria can be beneficial not only the tropical infection but also non - infectious tropical diseases. In a well-known tropical cancer, opisthorchiasis induced cholangiocarcinoma, the management of mitochondrial disorder is the new proposed therapeutic approach. Recently, Uchida et al. proposed that "The mitochondrial functionsupporting agent 1-carnitine is a good candidate to control oxidative stress conditions" in management of cholangiocarcinoma [5]

It is no doubt that the research on mitochondrial dysfunction in tropical diseases and development of new therapeutic approach focusing on mitochondria will be useful in tropical medicine.

\section{Conflict of interest}

None

\section{References}

1. Gorman GS, Chinnery PF, DiMauro S, Hirano M, et al. (2016) Mitochondrial diseases Nat Rev Dis Primers 2: 16080. [Crossref]

2. Zhang H, Feng YW, Yao YM (2018) Potential therapy strategy: Targeting mitochondrial dysfunction in sepsis. Mil Med Res 26: 5.

3. Goodman CD, Buchanan HD, McFadden GI (2017) Is the mitochondrion a good malaria drug target? Trends Parasitol 33: 185-193. [Crossref]

4. Lu MY, Liao F (2011) Interferon-stimulated gene ISG12b2 is localized to the inner mitochondrial membrane and mediates virus-induced cell death. Cell Death Differ 18: 925-936. [Crossref]

5. Uchida D, Takaki A, Ishikawa H, Tomono Y, Kato H, et al. (2016) Oxidative stress balance is dysregulated and represents an additional target for treating cholangiocarcinoma. Free Radic Res 50: 732-743. [Crossref]
Copyright: (C)2018 Wiwanitkit V. This is an open-access article distributed under the terms of the Creative Commons Attribution License, which permits unrestricted use, distribution, and reproduction in any medium, provided the original author and source are credited.
*Correspondence to: Viroj Wiwanitkit, Visiting Professor, Hainan Medical University, China, E-mail: wviroj@yahoo.com

Received: December 10, 2018; Accepted: December 17, 2018; Published: December 20, 2018 